\title{
Gravidanza e stress ossidativo
}

\author{
Giuliana Guido ${ }^{1}$, Roberta Talarico ${ }^{2}$, Maria Elena Donadio ${ }^{3}$, Santina Castellino ${ }^{4}$, \\ Rosanna Coppo ${ }^{3}$, Alessandro Amore ${ }^{3}$
}

\author{
${ }^{1}$ Nefrologia Dialisi, Policlinico Umberto I, Università "Sapienza" di Roma, Roma fellowship SIN 2012 \\ ${ }^{2}$ Nefrologia Dialisi, Policlinico Universitario "Magna Graecia" di Catanzaro, Catanzaro \\ ${ }^{3}$ Nefrologia Dialisi Trapianto, Policlinico Universitario Regina Margherita, Città della Salute e della Scienza di \\ Torino, Torino \\ ${ }^{4}$ UOC Nefrologia e Dialisi, Ospedale di Taormina, Taormina (ME)
}

\begin{abstract}
Pregnancy and oxidative STRess
Abstract. Pre-eclampsia is a relatively common complication of pregnancy, interesting about a $3 \%$ of the pregnancies. Its pathogenesis has been not yet completely clarified. However, a maternal endothelial dysfunction, particularly at the placenta level, seem to be the key factor in the development of this disease that clinically involves many organs such as kidney, brain and liver, characaterized by hypertension, proteinuria and oedema.

Recent works suggest a pathogenetic role of an altered expression of placental anti-angiogenic factors with consequent modifications in the redox state resulting in an oxidative stress. The effects to these anti-angiogenic factors results in a systemic endothelial dysfunction with hypertension, proteinuria, and the other systemic manifestations, such as encelophalopathy. Here, we will describe the most recent insights into the pathophysiology of preeclampsia attempting to provide a unifying hypothesis to reconcile the abnormalities at the feto-placental level and the clinical features of the maternal syndrome and provide a rationale for potential future prophylactic and therapeutic interventions for this pregnancy complication.
\end{abstract}

Key words: Pre-eclampsia, Pregnancy, Oxidative stress

Conflict of interest: None.

Ricevuto: 27 Maggio 2013; Accettato: 28 Maggio 2013

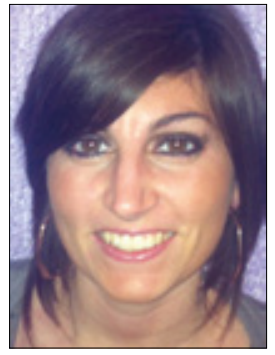

Giuliana Guido
La gravidanza come orchestrazione di differenti meccanismi

La conduzione di una gravidanza fisiologica senza complicanze sia materne che fetali è il risultato di una precisa orchestrazione tra diversi fattori, tra i quali un ruolo predominate viene svolto dai fattori genetici, influenzati a loro volta dai fattori ambientali e dai meccanismi di tolleranza immunitaria. A questi si aggiungono altri fattori che possono influenzare i meccanismi patogenetici: tra questi il comportamento sessuale, alcune abitudini e gli stili di vita.

Modificazioni di uno o più di questi fattori determinano l'insorgenza di complicanze durante la gravidanza, tra cui la preeclampsia, relativamente comune, che colpisce circa il $3 \%$ delle gestanti.

Il ruolo preciso dei fattori genetici nello sviluppo della preeclampsia non è stato del tutto chiarito e non è stato indivi- duato ancora nessun gene specifico. In un'analisi di linkage sono stati identificati 3 potenziali loci correlati a un'aumentata suscettibilità alla pre-eclampsia: 2p13, 2p25 e 9p13 $(1,2)$. Tuttavia, i risultati non sono ancora definitivi.

È da tenere in considerazione che fattori epigenetici possono modificare l'espressione di geni normali e amplificare gli effetti di polimorfismi che senza stimoli non risulterebbero fenotipicamente attivi.

Il tramite tra l'ambiente e il genoma è rappresentato dall'immunità innata, sistema di difesa filogeneticamente conservato nelle diverse specie. I recettori Toll (Toll like receptors, TLR) rappresentano il punto di congiunzione. Sono stati descritti sino a oggi 14 differenti TLR con specificità per diversi ligandi. Alcuni TLR, tra cui il 4 e il 2, sono espressi a livello della membrana citoplasmatica; altri, come il 3 , il 7 e il 9, sono intracitoplasmatici.

I ligandi dei TLR sono molteplici e, tra questi i lipopolisaccaridi batterici, il DNA, l'RNA, i PAMPS e i DAMPS (prodotti del catabolismo cellulare o batterico). È interessante osservare che, mentre il DNA e l'RNA umani se normometilati, non vengono riconosciuti dai TLR specifici, il DNA e l'RNA bat- 
terici vengono riconosciuti anche in condizione di normometilazione e come tali attivano il sistema dei TLR. Tra i target dei TLR, oltre a fattori trascrizionali, è di estrema importanza l'enzima DNA-dimetiltransferasi (DMTT) e la istone-deacetilasi. La DMTT ha un ruolo chiave nella trascrizione genica, in quanto demetila il DNA, condizione necessaria per l'attacco della DNA polimerasi e della RNA polimerasi. Ne risulta, pertanto, che fattori ambientali possono modificare l'espressione di geni e rendere fenotipicamente/funzionalmente importanti polimorfismi che, in condizioni di non stimolo, non avrebbero nessun significato biologico.

\section{Sistema immune e gravidanza}

La fecondazione dell'oocita da parte dello spermatozoo potrebbe innescare una serie di reazioni immunologiche che, se non controllate, porterebbero alla distribuzione dell'embrione. L'HLA paterno è potenzialmente riconosciuto come "non self" dal sistema immunitario materno, con la conseguente attivazione di una risposta immunologica e la distruzione dell'embrione nelle prime fasi della duplicazione cellulare.

Il mancato innesco di una reazione di alloriconoscimento e di attivazione $\mathrm{T}$ linfocitaria è legato all'instaurarsi di un fenomeno di "tolleranza immunitaria" con l'attivazione dei linfociti $\mathrm{T}$ regolatori ( $\mathrm{T}$ reg). L'esposizione ripetuta dell'HLA dello spermatozoo prima di una gravidanza sembrerebbe giocare un ruolo chiave nell'induzione della tolleranza immunitaria. Lo spermatozoo, infatti, contiene alte concentrazioni di TGF $\beta$, attivatore dei $\mathrm{T}$ reg. La tolleranza che si instaura non è, tuttavia, duratura nel tempo. Infatti, lunghi periodi intergravidici fanno sì che il fenomeno di tollerogenesi diventi inefficace con l'innesco di reazioni immuni materne contro l'embrione e il feto, non solo responsabili di aborti spontanei ma coinvolti anche nella patogenesi della pre-eclampsia (3-6).

\section{Pre-eclampsia}

La pre-eclampsia è una complicanza relativamente comune delle gravidanze e interessa circa il 3\% delle gestanti. È il risultato di uno squilibrio tra fattori placentari e materni (7) ed è una delle più frequenti cause di morbidità e mortalità fetale e materna. Clinicamente, si manifesta dopo la ventesima settimana di gestazione con proteinuria di diversa entità sino a valori nefrosici, ipertensione arteriosa ed edemi (8). È caratterizzata da un interessamento sistemico delle cellule endoteliali anche se ha come organi target più frequenti il rene, il fegato e l'encefalo.

La placenta svolge un ruolo centrale nello sviluppo della pre-eclampsia. Questa è infatti considerata come secondaria a un'alterazione della normale placentazione (7-9), fenomeno critico nel determinare il futuro decorso della gravidanza e la crescita del feto in assenza di complicanze. Nelle prime fasi che seguono l'impianto, la crescita embrionale ha un andamento esponenziale con spiccata proliferazione cellulare e conseguente elevato consumo energetico (elevate richieste di ossigeno e di ATP). Il numero di cellule prodotte durante la vita intrauterina supera largamente quello necessario per la formazione di un organismo definitivo fenotipicamente nor- male. Il rimodellamento dell'embrione e del feto durante la vita intrauterina sino al raggiungimento del fenotipo definitivo si ha grazie a fenomeni apoptotici. L'apoptosi è regolata da molti fattori tra cui l'angiotensina II, attraverso uno stimolo dei recettori ATII e i prodotti dei geni della famiglia PAX. Come è noto, l'apoptosi, o morte cellulare programmata, è una morte cellulare che non esita nel rilascio di componenti intracitoplasmatici, come le proteasi lisosomiali, e che non dà, a differenza della necrosi, esito a flogosi. Il rimodellamento apoptotico è finalizzato al raggiungimento di un fenotipo normale funzionalmente attivo del neonato e teleologicamente determinato dalla difesa della specie. A differenza della necrosi, che è passiva, l'apoptosi è un meccanismo attivo con sintesi di proteine ed enzimi e, quindi, ad elevato consumo energetico. È, pertanto, intuitivo che l'apporto di ossigeno e la sintesi mitocondriale di ATP siano elementi chiave nella conduzione di una gravidanza normale. La funzione della placenta è quella di fornire all'embrione, dopo il breve periodo di impiego delle scorte energetiche dello spermatozoo e dell'oocita, e al feto la quota necessaria di $\mathrm{O}_{2}$ e di fattori plastici (aminoacidi, glicidi, oligoelementi, ecc.). La placentazione è indotta dallo stato ipossico relativo. L'ipossia stimola il fattore inducibile dell'ipossia (Hypoxia Inducile Factor, HIF) attraverso la stimolazione di un enzima, la prolil-idrossilasi. Questo enzima distacca due radicali di prolina dall'HIF inattivo nel citoplasma e lo attiva rendendo possibile la sua traslocazione nucleare. L'HIF attivato passa nel nucleo e si lega a delle sequenze specifiche, note come hypoxia responsive genes, e ne induce la trascrizione. Tra i geni trascritti e tradotti implicati nei meccanismi di placentazione un ruolo principe è giocato dai geni codificanti per il vascular endothelial growth factor (VEGF) e il transforming growth factor $\beta$ (TGF- $\beta$ ). Il VEGF costituisce il più potente agente neo-angiogenetico noto, il cui effettore terminale è l'ossido nitrico (NO).

\section{Meccanismi fisiopatologici della placentazione}

In condizioni fisiologiche, i citotrofoblasti di origine fetale invadono le arterie spirali materne trasformandole in vasi capaci di fornire un'idonea perfusione, tale da garantire un'adeguata crescita fetale. Durante questo processo, i citotrofoblasti assumono un fenotipo endoteliale (pseudo-vasculogenesi) (1012). In questa fase prevalgono processi che favoriscono l'angiogenesi e, in particolare, gli attori principali sono il fattore VEGF, il Placental Growth Factor (PIGF) e il TGF- $\beta 1$ (11, 12). Durante una normale gravidanza, l'omeostasi vascolare è mantenuta dall'espressione di fattori pro-angiogenetici e antiangiogenetici. L'alterazione di questo delicato equilibrio porta all'innesco di meccanismi responsabili della pre-eclampsia (Fig. 1). In questa, infatti, i fattori ad attività anti-angiogenetica prevalgono su quelli pro-angiogenetici, portando ad alterazioni funzionali della placenta con la consequenziale riduzione dell'apporto di $\mathrm{O}_{2}$ al feto e il rilascio di fattori flogogeni. Fattori scatenanti il meccanismo pre-eclamptico sono la secrezione placentare di soluble fms-like Tyrosine Kinase-1 (sFlt1) e di soluble endoglin (sEng), proteine endogene circolanti, che inibiscono rispettivamente il VEGF e il TGF- $\beta 1$ (13). I risultati finali sono la riduzione del numero dei vasi placentari e 


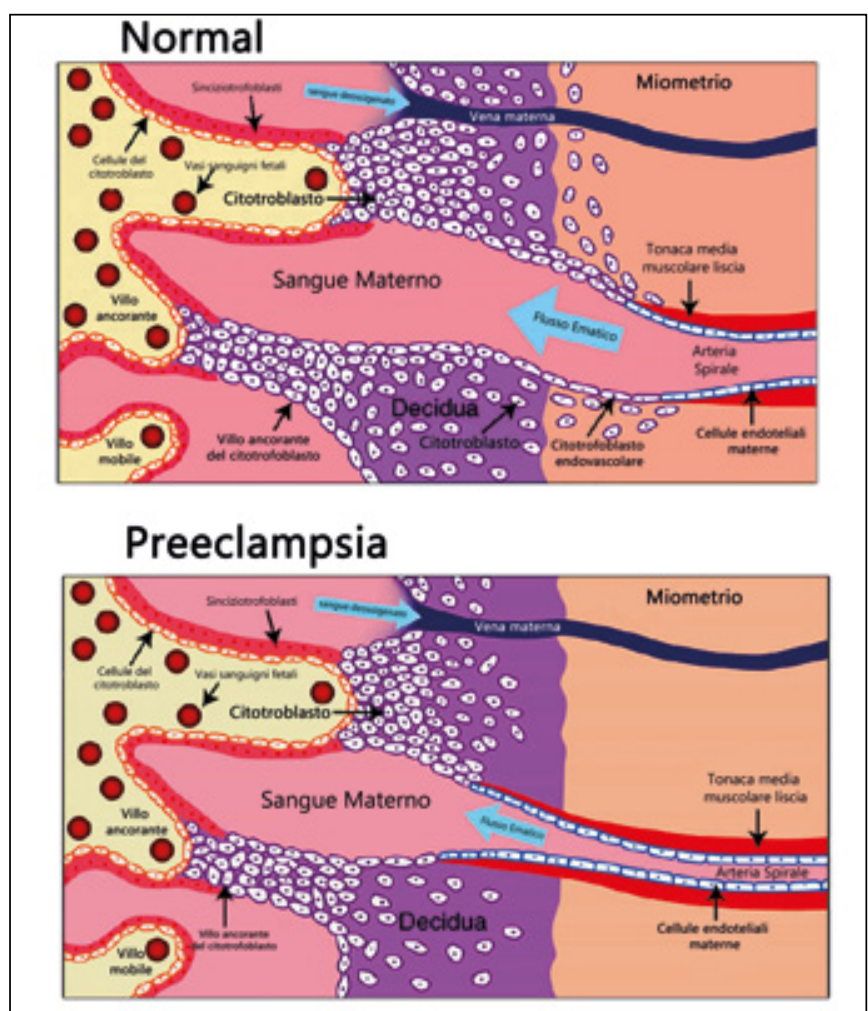

Fig. 1 - Le anomalie della placentazione nella pre-eclampsia. Durante il normale sviluppo placentare (in alto), $i$ citotrofoblasti di origine fetale invadono le arterie spirali materne trasformandole in vasi di calibro maggiore capaci di fornire un'adeguata perfusione placentare per sostenere la crescita fetale. Durante questo processo, $i$ citotrofoblasti assumono un fenotipo endoteliale (pseudovasculogenesi). Nella preeclampsia (in basso), $i$ citotrofoblasti non riescono ad assumere un fenotipo endoteliale. Inoltre, l'invasione delle arterie spirali è superficiale, pertanto rimangono vasi di piccolo calibro.

l'apporto di $\mathrm{O}_{2}$ ai tessuti fetali. Ne risulta una disfunzione endoteliale con la conseguente ridotta sintesi di prostacicline e di ossido nitrico. L'effetto terminale è la liberazione di fattori procoagulanti e vasocostrittori che amplificano il danno (14-18) (Fig. 2).

Nello sviluppo della pre-eclampsia, si possono schematicamente individuare tre stadi: i primi due, subclinici, sono determinati da una disfunzione della perfusione utero-placentare e dalla perdita di controllo della produzione di radicali liberi da parte delle cellule endoteliali alterate, responsabili di uno stato ossidativo feto-placentare. I radicali liberi, attraverso la stimolazione dei cosiddetti stress-sensitive pathway (NF-kB, Jun/Jak, MAPK, MAPK p38, ERK, ecc.) sensibili al $\mathrm{pH}$ locale, inducono una risposta infiammatoria responsabile dell'innesco dei fenomeni pre-eclamptici. Il terzo stadio, in cui la pre-eclampsia diventa clinicamente manifesta, è causato da un processo infiammatorio generalizzato con interessamento endoteliale sistemico in risposta al rilascio di fattori flogistici da parte della placenta danneggiata $(9,19)$.

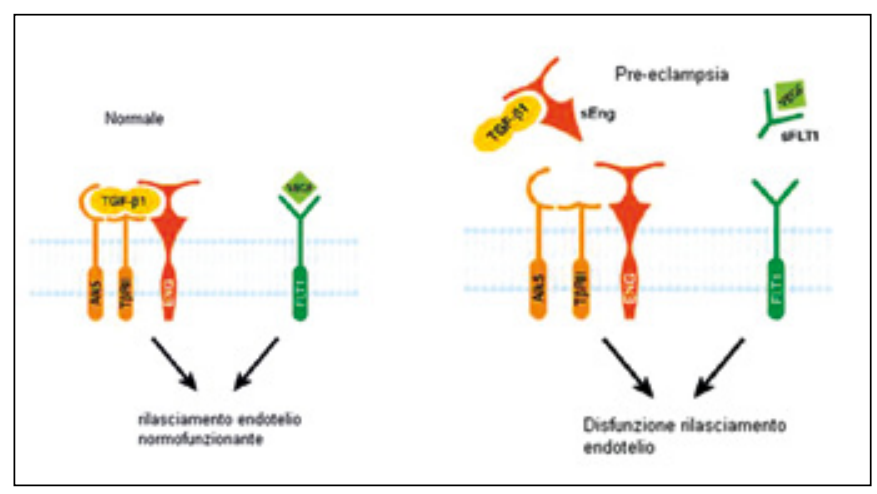

Fig. 2 - sFlt1 e sEng causano la disfunzione endoteliale antagonizzando l'effetto del VEGF e del TGF- $\beta 1$. VEGF e TGF- 11 sono necessari per il mantenimento del trofismo endoteliale in diversi tessuti, compresi il rene e, verosimilmente, la placenta. Durante la gravidanza normale, l'omeostasi vascolare è mantenuta da livelli fisiologici di VEGF e TGF- $\beta 1$ nel sistema vascolare. Nella pre-eclampsia, un'aumentata produzione placentare di sFlt1 e sEng inibisce l'attività di VEGF e TGF- $\beta 1$, con disfunzione endoteliale, ridotti livelli di prostaciclina e di ossido nitrico e aumentato rilascio di agenti procoagulanti.

\section{Stress ossidativo}

L'interazione tra VEGF e sFlt1 determina il rilascio di ossido nitrico (NO), che è un potente vasodilatatore (agisce sulla guanilato ciclasi, con sintesi finale di cGMP). L'NO è sintetizzato, a partire dall'arginina, dall' isoforma costitutiva endoteliale dell'ossido nitrico sintetasi (eNOS) (20).

La placenta umana è provvista solo della eNOS e non esprime la forma inducibile e neuronale dell'ossido nitrico sintetasi. Le sedi di sintesi e rilascio sono le cellule endoteliali del cordone ombelicale e i citotrofoblasti.

L'ossido nitrico ha, inoltre, un ruolo anche nella fase iniziale della gravidanza, quella dell'impianto dell'embrione, regolando l'espressione e l'attività delle Matrix-Degrading Proteases (MMP2 e MMP9) (21). Il rilascio di NO in concertazione con VEGF e sFlt1 è coinvolto nei meccanismi di invasione dei citotrofoblasti regolandone l'adesione alle caderine (molecole di adesione endoteliale) e, quindi, nei fenomeni neo-angiogenetici (22). Un'alterazione della sintesi/funzione dell'NO partecipa alla disfunzione placentare con l'innesco dei meccanismi che esitano nella pre-eclampsia e nell'eclampsia vera e propria.

Nel corso della pre-eclampsia, la diminuita attività dell’NO non è secondaria a una diminuita produzione. Infatti, le placente delle donne pre-eclamptiche esprimono normali livelli di eNOS e sintetizzano normali quantità di NO. Nelle donne pre-eclamptiche l'NO prodotto è instabile con un'emivita inferiore e facilmente degradabile. Essendo l'NO portatore di un elettrone spaiato è capace di interagire con altri radicali liberi, prodotti dallo stato ipossico, e di bloccare i gruppi prostatici di numerosi enzimi. L'interazione elettrica tra NO e altri radicali liberi esita nella formazione del perossinitrite $\left(\mathrm{ONOO}^{-}\right)$, un potente anione perossidante, citotossico e con proprietà antiangiogenetiche (23-25). 


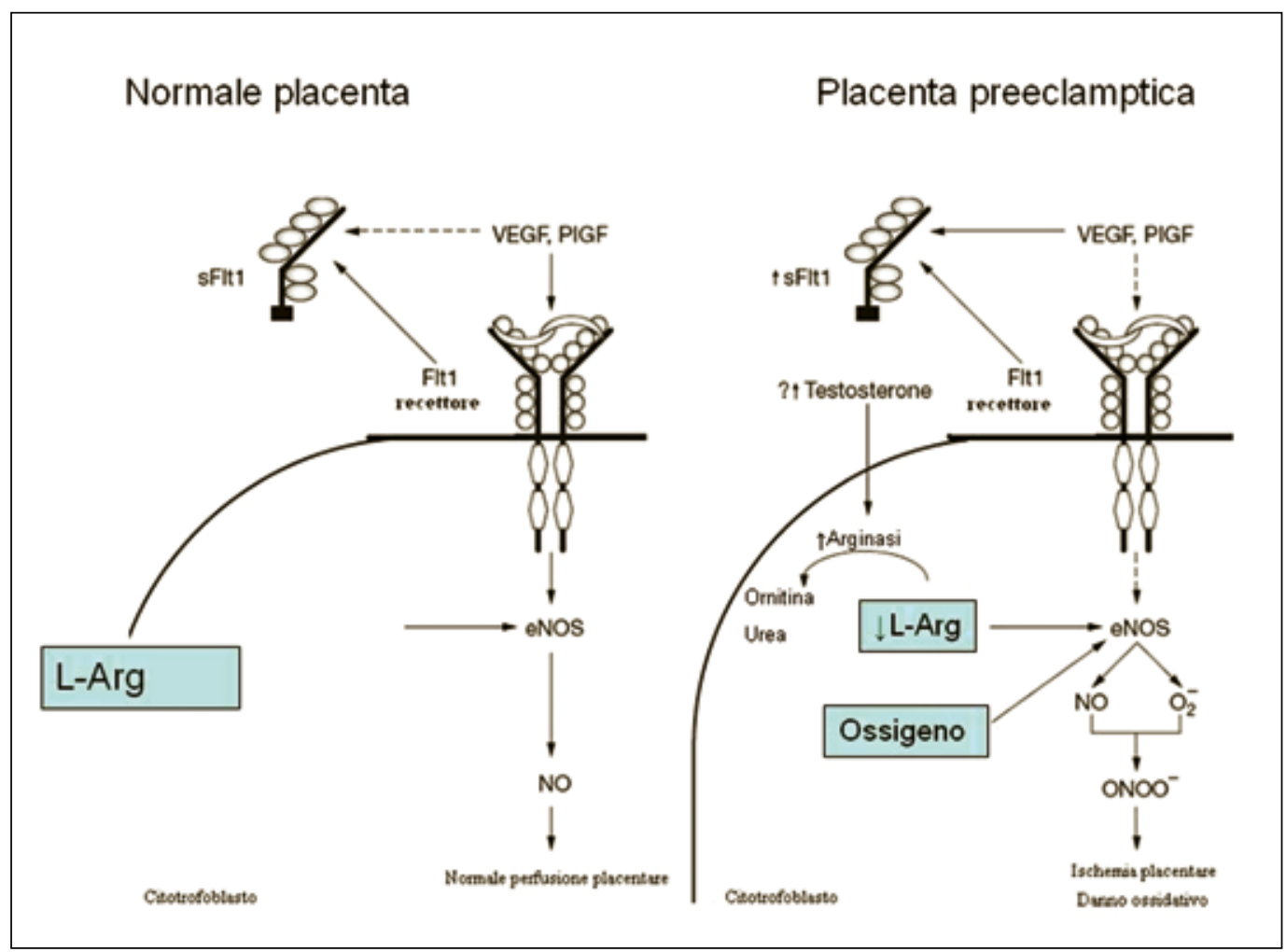

Fig. 3 - La deplezione di LArginina nella pre-eclampsia causa ipoperfusione placentare e danno microvascolare. Il legame di VEGF e PIGF al recettore di sFlt1 sui citotrofoblasti stimola la produzione di ossido nitrico da parte dell'ossido nitrico sintetasi endoteliale. L'interazione tra VEGF e PIGF col recettore di Flt1 è inibita da sFlt1. Una placenta normale, con produzione normale di arginina, permette un'adeguata sintesi di ossido nitrico endoteliale (pannello di sinistra). Nella pre-eclampsia, sFlt1 inibisce l'attivazione della sintetasi dell'ossido nitrico endoteliale, inducendo la formazione di anione superossido che, reagendo con l'NO, forma il perossinitrito. Si riduce, pertanto, l'emivita di NO (pannello di destra) con, di conseguenza, perfusione placentare anomala e danno ossidativo microvascolare.

L'alterata funzione dell'NO induce vasocostrizione dei vasi feto-placentari e, quindi, un'inadeguata perfusione con ipoperfusione placentare e ischemia. L'HIF, elevato nelle placente pre-eclamptiche, media l'adattamento cellulare a condizioni di ipossia, attraverso la trascrizione di diversi geni tra cui il TGF- $\beta 3$, potente inibitore della differenziazione dei trofoblasti $(12,26)$.

Inoltre, l'ipossia aumenta l'attività delle xantino-ossidasi implicate nella generazione dei ROS che contribuiscono alla disfunzione endoteliale materna (Fig. 3) (20, 27). I ROS modificano il metabolismo endoteliale attraverso il blocco del trasporto elettronico a livello mitocondriale, la perossidazione lipidica e l'ossidazione delle proteine. I lipidi perossidati e il perossinitrito svolgono una duplice azione: attivano le ciclossigenasi, con il conseguente incremento della sintesi di trombossani $\left(\mathrm{TxA}_{2}\right)$ con azione vasocostrittrice, e, contemporaneamente, inibiscono la sintesi delle prostacicline ( $\mathrm{PGI}_{2}$ ). Molto probabilmente questo disequilibrio $\mathrm{TxA}_{2}-\mathrm{PGI}_{2}$ precede l'esordio clinico della pre-eclampsia.

\section{Quadro clinico della pre-eclampsia}

I meccanismi patogenetici descritti rispecchiano il quadro clinico materno della pre-eclampsia, dominato dall'ipertensione arteriosa secondaria a uno squilibrio tra fattori vasodilatanti e vasocostrittori, a favore di questi ultimi. La proteinuria è secondaria a un danno podocitario. I fattori circolanti prodotti dalla placenta "pre-eclamptica" sortiscono un effetto sulle proteine podocitarie che partecipano alla formazione dello slit diaphragm. Il siero di pazienti pre-eclamptiche induce, in vitro, un'alterazione strutturale della cellula podocitaria, con modificazione della distribuzione della nefrina e con conseguenti alterazione del citoscheletro podocitario actinico e comparsa delle cosiddette stress fiber (28). La perdita della normale localizzazione di nefrina e della distribuzione spaziale dei filamenti actinici risulta nella perdita dello slit diaphragm, con l'appiattimento dei processi pedicillari e la conseguente comparsa di proteinuria.

\section{Stili di vita e sviluppo della pre-eclampsia}

Gli stili di vita possono contribuire allo sviluppo dell'alterazione dello stato ossido-riduttivo, che è alla base della preeclampsia. Non solo il fumo di sigaretta ma anche le abitudini alimentari influenzano lo stress ossidativo. La cottura dei cibi influenza molto il contenuto di prodotti avanzati della glicazione, i cosiddetti AGE (advanced glycated end products). Questi sono prodotti in condizioni di iperglicemia (reazione di Maillard), ma anche in condizioni di normoglicemia, in ambiente ossidato. Gli AGE si formano per una condensazione chetonica stabile tra gli $\varepsilon$-aminogruppi delle proteine e $\mathrm{i}$ radicali $\mathrm{OH}$ dei glicidi. Gli AGE sono resistenti alla proteolisi e possono depositarsi nei tessuti. Tuttavia, gli AGE non sono sostanze inerti, ma interagiscono con specifici recettori, RAGE, espressi su numerose linee cellulari, tra cui monocitimacrofagi, cellule endoteliali, renali e così via. L'attivazione dei RAGE da parte degli AGE attiva una cascata di trasmissione del messaggio del nucleo, con l'attivazione di numerosi fattori trascrizionali, tra cui NF-kB. L'esito finale è la sintesi di fattori flogistici vasocostrittori che partecipano al danno en- 
doteliale caratteristico della pre-eclampsia e lo amplificano. Sono noti due classi di RAGE, RAGE e AGR1 o Galectina 3. L'attivazione dei RAGE si estrinseca in un effetto ossidante e infiammatorio, quello degli AGR1 in un effetto antiossidante e antinfiammatorio. Nella pre-eclampsia è stata descritta una prevalenza dei RAGE (29).

\section{Terapia}

Numerose ricerche sulla fisiopatologia delle alterazioni placentari e materne nella pre-eclampsia costituiscono il razionale da cui partire per interventi terapeutici futuri.

La somministrazione di L-arginina dovrebbe teoricamente controbilanciare l'eccessiva degradazione dell'NO, con una minore produzione di perossinitrito.

A supportare tale ipotesi, dati preliminari in diversi trial recenti hanno dimostrato che la supplementazione di L-arginina è associata a una significativa riduzione della pressione arteriosa e a una diminuzione delle resistenze delle arterie uterine. Inoltre, la somministrazione di vitamine $\mathrm{C}$-E riduce gli indici di stress ossidativi e la frequenza della pre-eclampsia (30-32). Questi risultati indicano che lo stress ossidativo dovrebbe essere considerato il target per la prevenzione della preeclampsia.

\section{Conclusioni}

In conclusione, al momento attuale, sebbene i meccanismi fisiopatologici non siano ancora completamente chiariti, è accettato che la pre-eclampsia sia il risultato di una modificazione dei normali meccanismi di placentazione e che l'effettore finale del danno sia riconducibile a un'alterata attività dell'NO, verosimilmente secondaria a una ridotta biodisponibilità tissutale di L-arginina. Lo stato ossidativo causa la disfunzione endoteliale con l'innesco dei meccanismi preeclamptici. Il rilascio, da parte dell'endotelio placentare alterato, di fattori circolanti tra cui sFlt1 e radicali liberi indurrebbe una disfunzione endoteliale sistemica e lo sviluppo di uno stato eclamptico. Al momento, i livelli sierici di sFlt1 possono essere considerati come un marker di pre-eclampsia.

\section{Riassunto}

La pre-eclampsia è una complicanza relativamente comune della gravidanza, che interessa circa il 3\% delle gravidanze. Attualmente, la patogenesi non è stata ancora completamente chiarita.

Comunque, la disfunzione endoteliale materna, specialmente a livello della placenta, sembra essere il fattore chiave per lo sviluppo di questa malattia che clinicamente coinvolge molti organi, come reni, cervello e fegato, e che è caratterizzata da ipertensione, proteinuria ed edema.

Lavori recenti suggeriscono il ruolo patogenetico di un'alterata espressione di fattori anti-angiogenici placentari con conseguenti modifiche dello stato redox che provocano stress ossidativo.

La risposta all'azione dei fattori anti-angiogenetici produce una disfunzione endoteliale sistemica con ipertensione, proteinuria e altre manifestazioni sistemiche, come, per esempio, l'encefalopatia.

In questo articolo verranno descritte le conoscenze più recenti nella fisiopatologia della pre-eclampsia, cercando di dare un'ipotesi patogenetica comune per conciliare le anomalie a livello feto-placentare e le caratteristiche cliniche della sindrome materna e fornire una spiegazione logica per i potenziali futuri interventi profilattici e terapeutici di questa complicanza della gravidanza.

Parole chiave: Pre-eclampsia, Gravidanza, Stress ossidativo

Dichiarazione di conflitto di interesse: Gli Autori dichiarano di non avere conflitto di interessi.

\author{
Indirizzo degli Autori: \\ Dr.ssa Giuliana Guido \\ Nefrologia Dialisi \\ Policlinico Umberto I \\ Università "Sapienza" di Roma \\ 00185 Roma \\ giulianaguido83@gmail.com
}

\section{Bibliografia}

1. O'Shaughnessy K, Fu B, Ferraro F, et al. Factor V Leiden and thermolabile methylenetetrahydrofolate reductase gene variants in an East Anglian preeclampsia cohort. Hypertension 2009; 33: 1338-41.

2. Arngrímsson R, Sigurõardóttir S, Frigge ML, et al. A genomewide scan reveals a maternal susceptibility locus for preeclampsia on chromosome 2p13. Hum Mol Genet 1999; 8: 1799-805.

3. Billington WD. The immunological problem of pregnancy: 50 years with the hope of progress. A tribute to Peter Medawar. J
Reprod Immunol 2003; 60: 1-11.

4. Zhang J, Patel G. Partner change and perinatal outcomes: a systematic review. Paediatr Perinat Epidemiol 2007; 21 (Suppl. 1): 46-57.

5. Basso O, Christensen K, Olsen J. Higher risk of preeclampsia after change of partner. An effect of longer interpregnancy intervals? Epidemiology 2001; 12: 624-9.

6. Maitra U, Davis S, Reilly CM, Li L. Differential regulation of Foxp3 and IL-17 expression in CD4 T helper cells by IRAK-1. J Immunol 2009; 182: 5763-9.

7. Redman CW, Sargent IL. Immunology of Pre-Eclampsia. Am J 
Reprod Immunol 2010; 63 (6): 534-43.

8. Report of the National High Blood Pressure Education Program Working Group on High Blood Pressure in Pregnancy. Am J Obstet Gynecol 2000; 183 (1): S1-S22.

9. Burton GJ, Jauniaux E. Placental oxidative stress:from miscarriage to preeclampsia. J Soc Gynecol Investig 2004; 11: 342-52.

10. Zhou Y, Fisher SJ, Janatpour M, et al. Human cytotrophoblasts adopt a vascular phenotype as they differentiate. A strategy for successful endovascular invasion? J Clin Invest 1997; 99: 2139-51.

11. Zhou Y, McMaster M, Woo K, et al. Vascular endothelial growth factor ligands and receptors that regulate human cytotrophoblast survival are dysregulated in severe preeclampsia and hemolysis, elevated liver enzymes, and low platelets syndrome. Am J Pathol 2002; 160: 1405-23.

12. Caniggia I, Mostachfi H, Winter J, et al. Hypoxia-inducible factor-1 mediates the biological effects of oxygen on human trophoblast differentiation through TGFbeta(3). J Clin Invest 2000; 105: 577-87.

13. Maynard SE, Min JY, Merchan J, et al. Excess placental soluble fms-like tyrosine kinase 1 (sFlt1) may contribute to endothelial dysfunction, hypertension, and proteinuria in preeclampsia. J Clin Invest 2003; 111: 649-58.

14. Wathen KA, Tuutti E, Stenman UH, et al. Maternal serumsoluble vascular endothelial growth factor receptor-1 in early pregnancy ending in preeclampsia or intrauterine growth retardation. J Clin Endocrinol Metab 2006; 91: 180-4.

15. McKeeman GC, Ardill JE, Caldwell CM, et al. Soluble vascular endothelial growth factor receptor-1 (sFlt-1) is increased throughout gestation in patients who have preeclampsia develop. Am J Obstet Gynecol 2004; 191: 1240-6.

16. Levine RJ, Maynard SE, Qian C, et al. Circulating angiogenic factors and the risk of preeclampsia. N Engl J Med 2004; 350: 672-83.

17. Hertig A, Berkane N, Lefevre G, et al. Maternal serum sFlt1 concentration is an early and reliable predictive marker of preeclampsia. Clin Chem 2004; 50: 1702-3.

18. Maynard S, Epstein FH, Karumanchi SA. Preeclampsia and angiogenic imbalance. Annu Rev Med 2008; 59: 61-78.

19. Redman CW, Sargent IL. Placental stress and preeclampsia: a revised view. Placenta 2009; 30 (Suppl. A): S38-42.

20. Noris M, Perico N, Remuzzi G. Mechanisms of disease: Pre- eclampsia. Nat Clin Pract Nephrol 2005; 1 (2): 98-114.

21. Novaro V, Colman-Lerner A, Ortega FV, et al. Regulation of metalloproteinases by nitric oxide in humantrophoblast cells in culture. Reprod Fertil Dev 2001; 13: 411-20.

22. Chang CC, Chang TY, Yu CH, Tsai ML. Induction of VEcadherin in rat placental trophoblasts by VEGF through a NOdependent pathway. Placenta 2005; 26: 234-41.

23. Martin D, Conrad KP. Expression of endothelial nitric oxide synthase by extravillous trophoblast cells in the human placenta. Placenta 2000; 21: 23-31.

24. Orange SJ, Painter D, Horvath J, Yu B, Trent R, Hennessy A. Placental endothelial nitric oxide synthase localization and expression in normal human pregnancy and pre-eclampsia. Clin Exp Pharmacol Physiol 2003; 30: 376-81.

25. Davison JM, Homuth V, Jeyabalan A, et al. New aspects in the pathophysiology of preeclampsia. J Am Soc Nephrol 2004; 15 : 2440-8.

26. Rajakumar A, Brandon HM, Daftary A, Ness R, Conrad KP. Evidence of the functional activity of hypoxia-inducible transcription factors overexpressed in preeclamptic placentae. Placenta 2004; $25: 763-9$.

27. Parks DA, Williams TK, Beckman JS. Conversion of xanthine dehydrogenase to oxidase in ischemic rat intestine: a reevaluation. Am J Physiol 1988; 254: G768-74.

28. Collino F, Bussolati B, Gerbaudo E, et al. Preeclamtic sera induce nephrin shedding from podocytes through endothelin-1 release by endothelial glomerular cells. Am J Renal Physiol 2008;294(5):F1185-94.

29. Vlassara H, Torreggiani M, Post JB, Zheng F, Uribarri J, Striker GE. Role of oxidants/inflammation in declining renal function in chronic kidney disease and normal aging. Kidney Int Suppl. 2009;(114):S3-11.

30. Chappell L, Seed PT, Briley AL, et al.Effect of antioxidants on the occurrence of pre-eclampsia in women at increased risk: a randomized trial. Lancet 1999;354: 810-6.

31. Facchinetti F, Longo M, Piccinini F, Neri I, Volpe A. L-Arginine infusion reduces blood pressure in preeclamptic women through nitric oxide release.J Soc Gynecol Invest 1999;6: 202-7.

32. Chappell L, Seed PT, Kelly FJ, et al. Vitamin C and E supplementation in women at risk of preeclampsia is associated with changes in indices of oxidative stress and placental function. Am J Obstet Gynecol 2002;187(3): 777-84. 